\title{
On the Prediction of Strength from Hardness for Copper Alloys
}

\author{
S. Chenna Krishna, Narendra Kumar Gangwar, Abhay K. Jha, and Bhanu Pant
}

Materials Processing and Characterization Group, Vikram Sarabhai Space Centre, Trivandrum 695 022, India

Correspondence should be addressed to S. Chenna Krishna; chenna.sk@gmail.com

Received 23 November 2012; Revised 27 February 2013; Accepted 18 March 2013

Academic Editor: Steve Bull

Copyright (C) 2013 S. Chenna Krishna et al. This is an open access article distributed under the Creative Commons Attribution License, which permits unrestricted use, distribution, and reproduction in any medium, provided the original work is properly cited.

Hardness and strength values of over 55 copper alloys strengthened by solid solution strengthening, precipitation hardening, cold working, and dispersion strengthening were compiled. The yield strength (YS) and ultimate tensile strength (UTS) values of the copper alloys examined ranged between 50 to $1300 \mathrm{MPa}$ and 200 to $1400 \mathrm{MPa}$, respectively. The compiled values were classified based on strain-hardening potential an indirect method to understand the effect of strain-hardening characteristics. Least squares regression analysis was employed to establish correlations between strength and Vickers hardness values. Strain-hardening potential showed a significant effect on the correlations. In all the cases, a linear relation was obtained for both YS and UTS with hardness for the entire range of values under analysis. Simple empirical equations were proposed to estimate the strength using bulk hardness. The proposed correlations obtained for the entire range of values were verified with experimental values. A good agreement was observed between experimental and predicted values.

\section{Introduction}

Ever since indentation hardness testing has come into existence, there were studies to estimate other mechanical properties especially ultimate tensile strength and yield strength from bulk hardness measurement [1-3]. Over the last 70 years, a number of researchers have worked on experimental techniques and theoretical relations to determine the strength from hardness. Such relations can be useful in the design, where direct measurement of tensile properties is not viable. These relations are always attractive as they bring down the number of tests to be conducted to ensure the quality of the materials $[4,5]$. As these methods are fast and relatively non-destructive in nature they are effectively used in failure analysis $[5,6]$.

Tabor [1] has shown that the ratio of ultimate tensile strength to the Vickers hardness is related to strainhardening coefficient. The relation between these parameters was expressed using

$$
\mathrm{UTS}=\left(\frac{H}{C}\right)(1-n)\left(\frac{12.5 n}{(1-n)}\right)^{n}
$$

where UTS is the ultimate tensile strength, $H$ is the Vickers hardness, $n$ is the strain-hardening coefficient, and $C$ is a constant which has a value of 2.9 for steels and 3.0 for copper alloys.

This relation was further improved by Cahoon [2] in the form of

$$
\mathrm{UTS}=\left(\frac{H}{2.9}\right)\left(\frac{n}{0.217}\right)^{n}
$$

where $H$ is the Vickers hardness and $n$ is the strain-hardening coefficient.

Cahoon et al. [3] proposed relation to estimate the yield strength using simple hardness measurement for copper, aluminum, and steel in the form of

$$
\mathrm{YS}=\left(\frac{H}{3}\right)\left(\frac{1}{10}\right)^{n}
$$

All the expressions mentioned previously require the knowledge of strain-hardening coefficient, which can be directly determined from uniaxial tensile or compression test and indirectly through Meyers hardness measurement and empirical relations [4]. Apart from these, few methods were proposed for estimation of strength for metals using an indentation technique in the literature [4-9].

Pavlina and Van Tyne [10] suggested simple linear relationships to estimate the ultimate tensile strength and yield 
strength using the Vickers hardness number for steels as follows:

$$
\begin{gathered}
\mathrm{YS}=-90.7+2.876 \mathrm{Hv}, \\
\mathrm{UTS}=-99.8+3.734 \mathrm{Hv},
\end{gathered}
$$

where strength has units of $\mathrm{MPa}$ and $\mathrm{Hv}$ has units of $\mathrm{kg} / \mathrm{mm}^{2}$. These relationships do not require the knowledge of any other parameter than hardness for estimation of strength.

Even though extensive work was conducted to estimate the ultimate tensile strength and yield strength from hardness measurements for certain metals and alloys, there appears that no attempts were made to obtain such relations for copper alloys. Most of the correlations available were obtained by analysis of data for steels [1-10]. Therefore, an attempt has been made to propose correlations to estimate the strength from hardness for copper alloys.

\section{Materials and Testing}

In the present investigation, hardness and strength values for copper alloys were collected from the literature. For comparison, few hardness values were converted from $\mathrm{HB}$ and $\mathrm{HRB}$ to Vickers hardness as per ASTM E140-07 [11]. All the hardness and strength values compiled were divided based on strainhardening potential as low, medium, and high UTS/YS ratios. Least squares regression analysis was employed to obtain simple expressions, to predict the strength from hardness.

In addition to the literature values, hardness and strength were evaluated for some copper alloys for validation of empirical relations obtained. These measurements were made in different heat treatment, cold-worked, and hot-worked conditions. Samples for hardness were ground and polished with emery paper prior to hardness measurement. Seven readings were taken for each condition and average is reported. The hardness was measured using a Vickers hardness tester (make: FIE FE-20) with a load of $10 \mathrm{kgf}$. Tensile properties were evaluated with dog-bone shaped flat tensile specimens ( $25 \mathrm{~mm}$ gauge length) using an INSTRON 5500R UTM at a strain rate of $10^{-3} \mathrm{~s}^{-1}$. Three tests were performed for each condition and average is reported.

\section{Results}

3.1. Entire Data Range. The relationship between compiled ultimate tensile strength and hardness values (120 data points) for different copper alloys is shown in Figure 1. A linear correlation could be observed in most of the hardness ranges of materials tested, except at low hardness. The linear trend line is made to pass through the origin as shown in Figure 1. Regression analysis of the data points yielded a linear relation as

$$
\mathrm{UTS}=3.353 * \mathrm{VHN}
$$

where ultimate tensile strength is in $\mathrm{MPa}$ and VHN is Vickers hardness number. Regression analysis for (6) gives a coefficient of determination $\left(R^{2}\right)$ of 0.933 . Even though

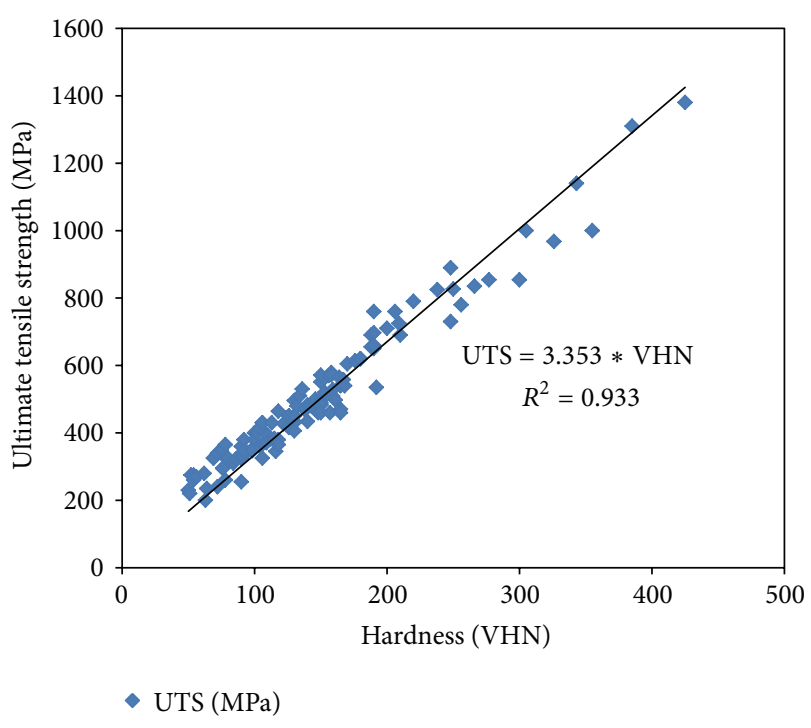

Figure 1: Correlation between hardness and ultimate tensile strength for copper alloys [12-28].

a linear correlation has been obtained from the entire range of the hardness, a deviation was observed for hardness lower than $110 \mathrm{VHN}$.

Correlation obtained for yield strength and hardness values for copper alloys also depicted a linear relationship as shown in Figure 2. Regression analysis of the data points gave a linear correlation for ultimate tensile strength as

$$
\mathrm{YS}=2.874 * \mathrm{VHN}
$$

where YS is in $\mathrm{MPa}$ and VHN is Vickers hardness number. Coefficient of determination $\left(R^{2}\right)$ for $(7)$ has a value of 0.916 . From Figure 2, it can be ascertained that yield strength has a linear correlation with hardness, with correlation tending to nonlinearity at hardness values less than $110 \mathrm{VHN}$.

3.2. Data Classified by UTS to YS Ratio. Since the compiled data taken from the literature comprises a large number of alloys, strain-hardening coefficient could not be obtained for all the alloys. Therefore, strain-hardening potential determined as the ratio of UTS to YS, which is a measure of maximum strengthening that can occur in a material beyond its yield strength and continues until UTS, was used in the study [10]. The ratio of these two parameters (UTS/YS) was employed to study the effect of strain-hardening on the material indirectly. To determine the effect of strain hardening on the compiled values, the data were classified into three groups: (i) low UTS/YS ratio (UTS/YS $<1.20$ ), (ii) medium UTS/YS ratio $(1.20<\mathrm{UTS} / \mathrm{YS}<1.52)$, and (iii) high UTS/YS ratio (UTS/YS > 1.52). Figures 3(a)-3(c) show the plots of strength and hardness values with the trend line obtained by regression analysis. The values of the regression coefficient and $R^{2}$ are given in Table 1 . Even though the data were fitted by a linear equation for all three plots in Figure 3, a variation was observed in the values of $R^{2}$. It is noteworthy to mention that best fit was observed in the case of low UTS/YS 
TABLE 1: Results of regression analysis for hardness-strength data of alloys.

\begin{tabular}{lccccc}
\hline Data type & Property & Regression equation & $R^{2}$ & Valid range (VHN) & Data points \\
\hline All & YS & YS $=2.874 *$ VHN & 0.916 & $50-425$ & $50-425$ \\
& UTS & UTS $=3.353 *$ VHN & 0.933 & $50-220$ & 120 \\
Medium & YS & YS $=2.594 *$ VHN & 0.887 & $50-220$ & 30 \\
UTS/YS & UTS & UTS $=3.389 *$ VHN & 0.934 & $50-142$ & $50-142$ \\
High & YS & YS $=1.97 *$ VHN & 0.845 & $78-425$ & $78-425$ \\
UTS/YS & UTS & UTS $=3.865 *$ VHN & 0.665 & 0.933 & 64 \\
Low & YS & YS $=3.018 *$ VHN & 0.917 & 64 \\
UTS/YS & UTS & UTS $=3.301 *$ VHN & & 78 \\
\hline
\end{tabular}

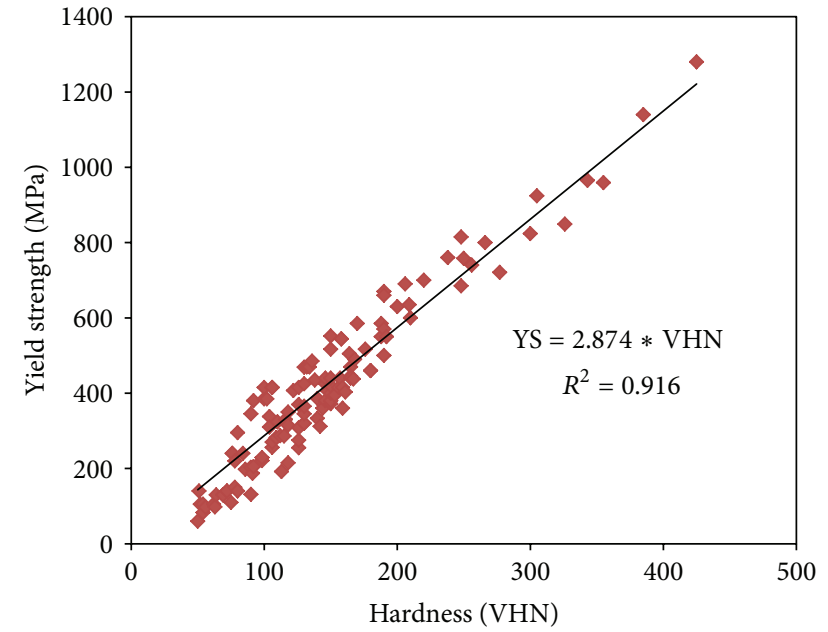

- YS (MPa)

Figure 2: Correlation between hardness and yield strength for copper alloys [12-28].

ratio with a coefficient of determination of 0.933 and 0.917 for UTS-hardness and YS-hardness, respectively.

\section{Discussion}

The results of the regression analysis obtained for different data groups are given in Table 1 . It is seen that all the data analyzed could be modelled by linear equations. However, some deviation from the linear trend line was observed in all the cases as shown in Figures 1-3. This can be attributed to the fact that a wide range of copper alloys with different thermomechanical and thermal history were analyzed in the present work. In addition, it may be due to the fact that the hardness measurement is done on samples with varying surface roughness using different indentation load, as both affect the measured hardness. It is noteworthy to mention that the lower range of hardness showed a deviation from linearity for both yield strength and ultimate tensile strength in the data groups analyzed as shown in Figures 1-3. It can be observed that UTS-hardness showed better correlation with $R^{2}$ value of 0.9333 with regression constant of 3.05. On the other hand, YS-hardness showed a regression coefficient and coefficient of determination of 2.874 and 0.916 , respectively. The regression coefficient obtained for copper alloys in the case of YS-hardness is similar to that reported for steels with a value of 2.876 [10]. Zinkle et al. [23] proposed linear correaltions for AMZIRC and AMAX-MZC between YS and hardness with regression constant of 3.03 and 3.0, respectively.

Empirical relations obtained for hardness strength classified by strain-hardening potential showed a significant effect on the correlations obtained for the data as evident from Table 1. Copper alloys with high strain-hardening potential showed a large deviation from the linear trend line for both YS and UTS as shown in Figure 3(a). This data group to a larger extent consists of alloys in solution treated condition and solid solution strengthened alloys in annealed condition. The hardness values of the alloy analyzed for this group were in the range of 50-140 VHN. On the other hand, data groups with with medium and low UTS/YS showed better correlations for strength hardness with minimal deviations from the linear trend line as shown in Figures 3(b) and 3(c). Among the three data groups analyzed, low UTS/YS group showed the best correlation with regression coefficient of 3.301 and 3.018 for UTS-hardness and YS-hardness, respectively. From the plot, it is also apparent that both trend lines lie very close and have similar behavior. This data group consists alloys that are subjected to cryorolling, cold rolling, and cold rolling + aging. The observed effect of strain hardening on the correlations may be due to wide differences in cold-working that occurs before hardness testing and during the test itself for annealed alloys. An annealed material with high strain-hardening potential will harden much more during the hardness test than a cold-worked metal. Therefore, cold-worked materials yield better correlations for hardness strength.

The predicted values of UTS and YS from hardness using (6) and (7) are compared with the experimental values as shown in Figure 4 . The predicted values are plotted as a line and experimental values are indicated by symbols. It can be seen that a reasonably good agreement is observed between experimental and predicted values for UTS whereas, experimental YS showed a reasonable deviation from predicted values at hardness lower than 120 VHN. Nevertheless, the experimental UTS values showed deviation at higher hardness as evident from Figure 4. It was observed that 


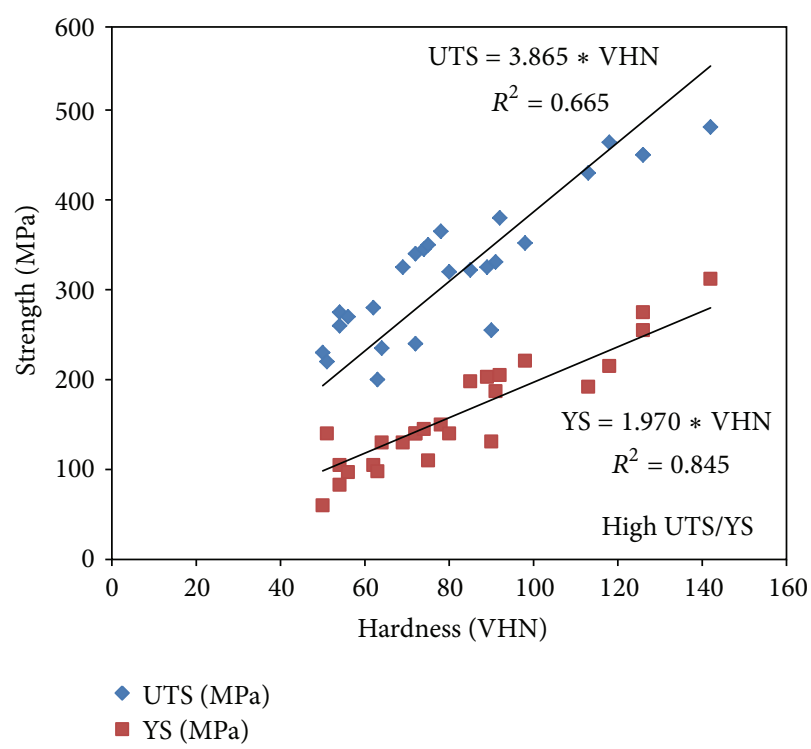

(a)

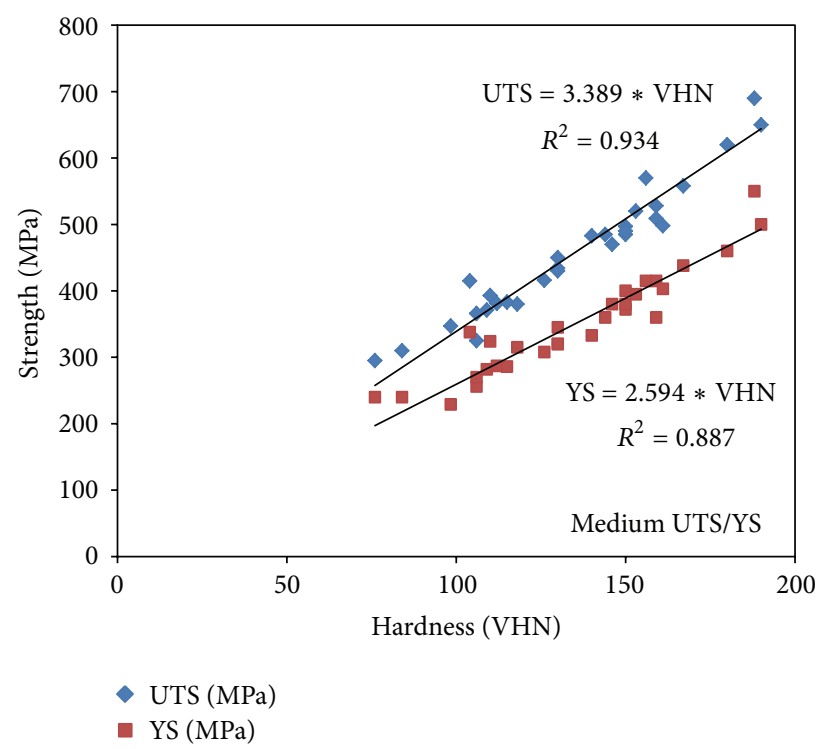

(b)

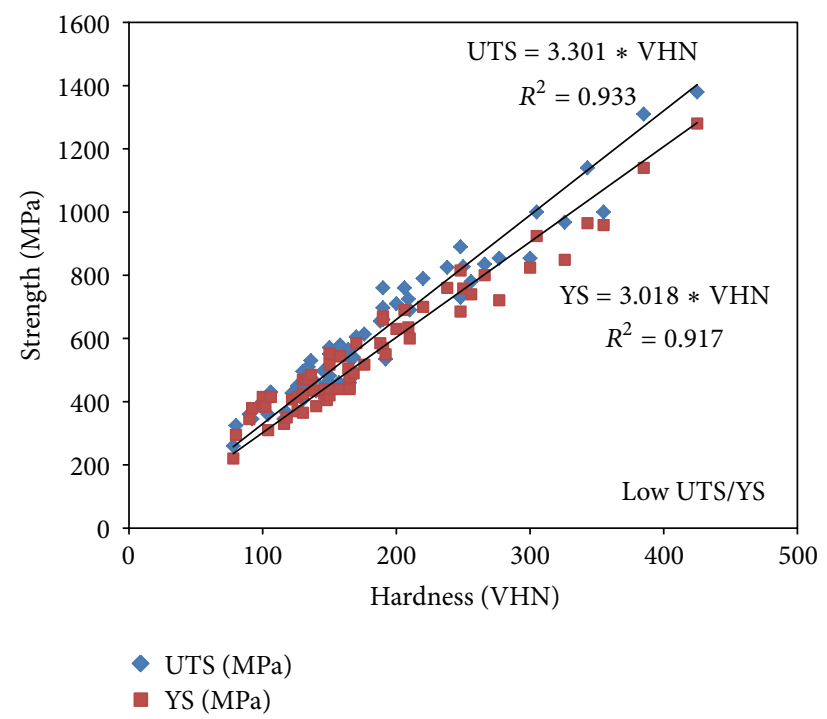

(c)

FIGURE 3: Relationship between strength and hardness of various alloys: (a) high UTS/YS ratio, (b) medium UTS/YS ratio, and (c) low UTS/YS ratio [12-28].

(6) estimated the UTS with less than 20\% deviation at hardness lower than $150 \mathrm{VHN}$. Whereas reasonable deviation was observed at higher hardness. On the other hand, YS was estimated within 10\%-25\% using (7) with large deviation at hardness lower than $140 \mathrm{VHN}$. These deviations are quite reasonable considering the inherent errors in measuring the hardness and strength [3]. Hence, the correlations can be utilized to estimate the strength of copper alloys from Vickers hardness.

\section{Conclusion}

The results of the present study indicate that yield strength and ultimate tensile strength of the copper alloys can be determined with fairly good precision from Vickers hardness using simple linear correlations. However, the strength can be estimated with better accuracy using correlations for alloys with low and medium strain-hardening potential, that is, for fully cold-worked, thermomechanically treated, and aged alloys. Alloys with lower hardness and strength have shown deviation from linear correlation. The equations proposed for all the data were validated with the experimental data and a good agreement was noticed for both yield strength and ultimate tensile strength. Therefore, it is concluded that these correlations can be used for predicting the strength of copper alloys covering a wide range (YS-50 to $1200 \mathrm{MPa}$ and UTS-200 to 1400 $\mathrm{MPa})$. 


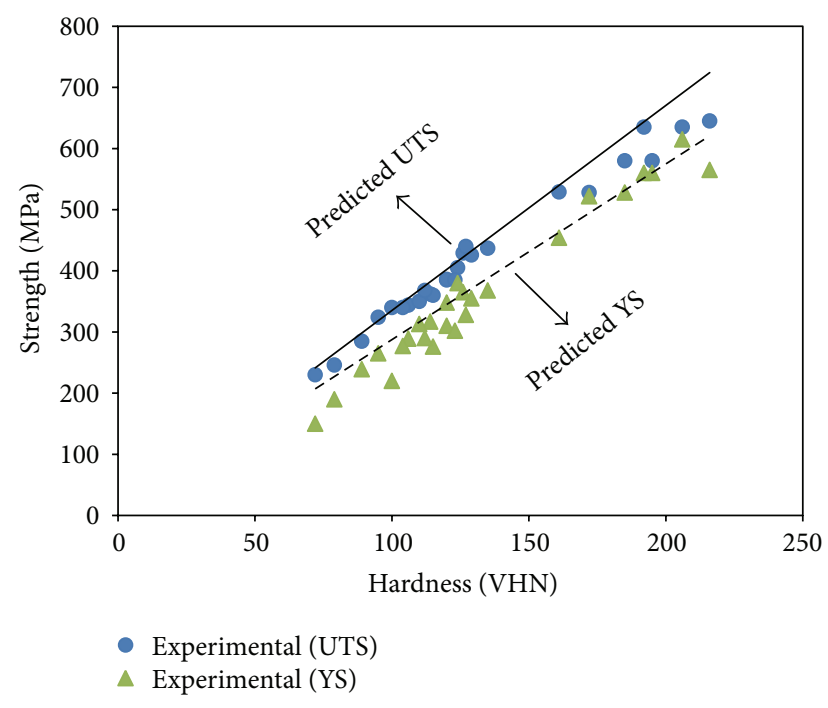

Figure 4: Comparison of estimated strength ((6) and (7)) and experimental strength for copper alloys.

\section{Acknowledgments}

The authors would like to thank Group Director (MMG) and Deputy Director VSSC (MME) for their support and technical guidance. The authors would like to express sincere gratitude to the Director, VSCC, for his kind permission to publish this work.

\section{References}

[1] D. Tabor, "The hardness and strength of metals," Journal Institute of Metals, vol. 79, pp. 1-18, 1951.

[2] J. R. Cahoon, "An improved equation relating hardness to ultimate strength," Metallurgical Transactions, vol. 3, no. 11, p. 3040, 1972.

[3] J. R. Cahoon, W. H. Broughton, and A. R. Kutzak, "The determination of yield strength from hardness measurements," Metallurgical Transactions, vol. 2, no. 7, pp. 1979-1983, 1971.

[4] J. Moteff, R. K. Bhargava, and W. L. McCullough, "Correlation of the hot-hardness with the tensile strength of 304 stainless steel to temperatures of $1200 \circ C$," Metallurgical Transactions A, vol. 6 , no. 5, pp. 1101-1104, 1975.

[5] M. O. Lai and K. B. Lim, "On the prediction of tensile properties from hardness tests," Journal of Materials Science, vol. 26, no. 8, pp. 2031-2036, 1991.

[6] S. C. Chang, M. T. Jahn, C. M. Wan, J. Y. M. Lee, and T. K. Hsu, "The determination of tensile properties from hardness measurements for Al-Zn-Mg alloys," Journal of Materials Science, vol. 11, no. 4, pp. 623-630, 1976.

[7] J. Datsko, L. Hartwig, and B. McClory, "On the tensile strength and hardness relation for metals," Journal of Materials Engineering and Performance, vol. 10, no. 6, pp. 718-722, 2001.

[8] E. G. Herbert, W. C. Oliver, and G. M. Pharr, "On the measurement of yield strength by spherical indentation," Philosophical Magazine, vol. 86, no. 33-35, pp. 5521-5539, 2006.

[9] G. Pintaude, A. Hoechele, and G. Cipriano, "Relation between strain hardening exponent of metals and residual profiles of deep spherical indentation," Materials Science and Technology, vol. 28, no. 9-10, pp. 9-10, 2012.

[10] E. J. Pavlina and C. J. Van Tyne, "Correlation of Yield strength and Tensile strength with hardness for steels," Journal of Materials Engineering and Performance, vol. 17, no. 6, pp. 888-893, 2008.

[11] Annual Book of ASTM Standards, Standard Hardness Conversion Tables for Metals Relationship among Brinell Hardness, Vickers Hardness, Rockwell Hardness, Superficial Hardness, Knoop Hardness, and Scleroscope, E140-07, American Society for Testing and Materials, West Conshohocken, Pa, USA, 2007.

[12] S. C. Krishna, K. Thomas Tharian, B. Pant, and R. S. Kottada, "Age-hardening characteristics of Cu-3Ag- $0.5 \mathrm{Zr}$ alloy," Materials Science Forum, vol. 710, pp. 563-568, 2012.

[13] I. S. Batra, G. K. Dey, U. D. Kulkarni, and S. Banerjee, "Microstructure and properties of a $\mathrm{Cu}-\mathrm{Cr}-\mathrm{Zr}$ alloy," Journal of Nuclear Materials, vol. 299, no. 2, pp. 91-100, 2001.

[14] H. Ehsanian Mofrad, S. Raygan, B. Amin Forghani, K. Hanaei, and F. K. Ahadi, "Effect of cold-working and aging processes on the microstructure, mechanical properties and electrical conductivity of $\mathrm{Cu}-13.5 \% \mathrm{Mn}-4 \% \mathrm{Ni}-1$. 2\%Ti alloy," Materials and Design, vol. 41, no. 0, pp. 182-191, 2012.

[15] S. C. Krishna, K. Radhika, K. T. Tharian, G. S. Rao, M. S. Kiranmayee, and B. Pant, "Effect of simulated brazing cycle on the microstructure and mechanical properties of $\mathrm{Cu}-\mathrm{Cr}-\mathrm{Zr}-\mathrm{Ti}$ alloy," Materials Science Forum, vol. 710, pp. 626-631, 2012.

[16] R. Markandeya, S. Nagarjuna, and D. S. Sarma, "Precipitation hardening of Cu-Ti-Cr alloys," Materials Science and Engineering A, vol. 371, no. 1-2, pp. 291-305, 2004.

[17] S. Nagarjuna, U. Chinta Babu, and P. Ghosal, "Effect of cryorolling on age hardening of $\mathrm{Cu}-1.5 \mathrm{Ti}$ alloy," Materials Science and Engineering A, vol. 491, no. 1-2, pp. 331-337, 2008.

[18] K. Oishi, I. Sasaki, and J. Otani, "Effect of silicon addition on grain refinement of copper alloys," Materials Letters, vol. 57, no. 15, pp. 2280-2286, 2003.

[19] B. N. Singh, D. J. Edwards, and P. Toft, "Effects of neutron irradiation on mechanical properties and microstructures of dispersion and precipitation hardened copper alloys," Journal of Nuclear Materials, vol. 238, no. 2-3, pp. 244-259, 1996.

[20] A. Vinogradov, T. Ishida, K. Kitagawa, and V. I. Kopylov, "Effect of strain path on structure and mechanical behavior of ultrafine grain $\mathrm{Cu}-\mathrm{Cr}$ alloy produced by equal-channel angular pressing," Acta Materialia, vol. 53, no. 8, pp. 2181-2192, 2005.

[21] R. Markandeya, S. Nagarjuna, and D. S. Sarma, "Effect of prior cold work on age hardening of $\mathrm{Cu}-3 \mathrm{Ti}-1 \mathrm{Cr}$ alloy," Materials Characterization, vol. 57, no. 4-5, pp. 348-357, 2006.

[22] R. Markandeya, S. Nagarjuna, and D. S. Sarma, "Characterization of prior cold worked and age hardened Cu-3Ti-1Cd alloy," Materials Characterization, vol. 54, no. 4-5, pp. 360-369, 2005.

[23] S. J. Zinkle, D. H. Plantz, A. E. Bair, R. A. Dodd, and G. L. Kulcinski, "Correlation of the yield strength and mlcrohardnesss of high-strength, high-conductivity copper alloys," Journal of Nuclear Materials C, vol. 133-134, pp. 685-689, 1985.

[24] A. Guha, "Development a high strength high conductivity $\mathrm{Cu}$ Ni-Be alloy," in High Conductivity Copper and Aluminum Alloys, E. Ling and W. Pierre, Eds., pp. 133-145, The Metallurgical Society of AIME, California, Calif, USA, 1984.

[25] S. C. Krishna, J. Srinath, A. Jha, B. Pant, S. C. Sharma, and K. George, "Microstructure and properties of a high-strength $\mathrm{Cu}-\mathrm{Ni}-\mathrm{Si}-\mathrm{Co}-\mathrm{Zr}$ alloy," Journal of Materials Engineering and Performance, 2013. 
[26] F. R. Mollar, K. G. Wikle, and A. R. Chaudhry, "CopperBeryllium for elevated temperature electronic applications," in High Conductivity Copper and Aluminum Alloys, E. Ling and W. Pierre, Eds., pp. 147-167, The Metallurgical Society of AIME, California, Calif, USA, 1984.

[27] ASM International Handbook Committee, ASM Handbook, Volume 02-Properties and Selection: Nonferrous Alloys and Special-Purpose Materials, ASM International, California, Calif, USA, 1990.

[28] E. G. West, Copper and Its Alloys, Ellis Haorwood Limited, Chichester, UK, 1983. 

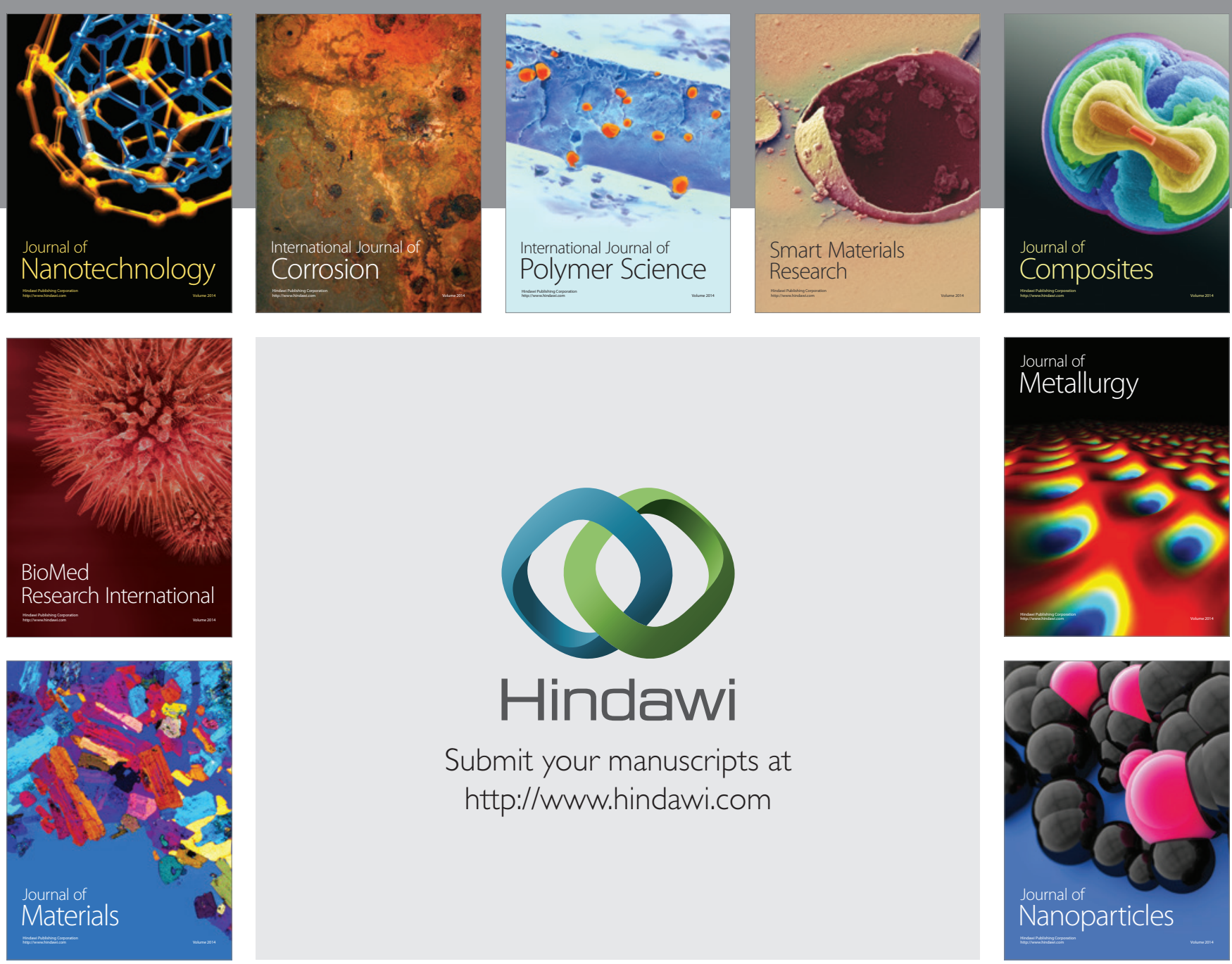

Submit your manuscripts at http://www.hindawi.com
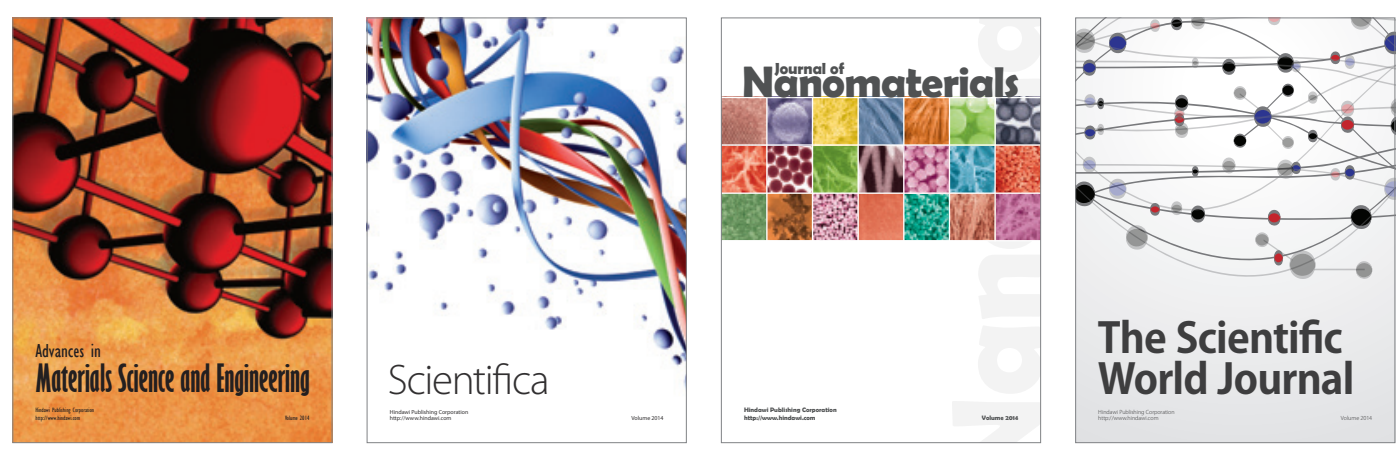

\section{The Scientific World Journal}
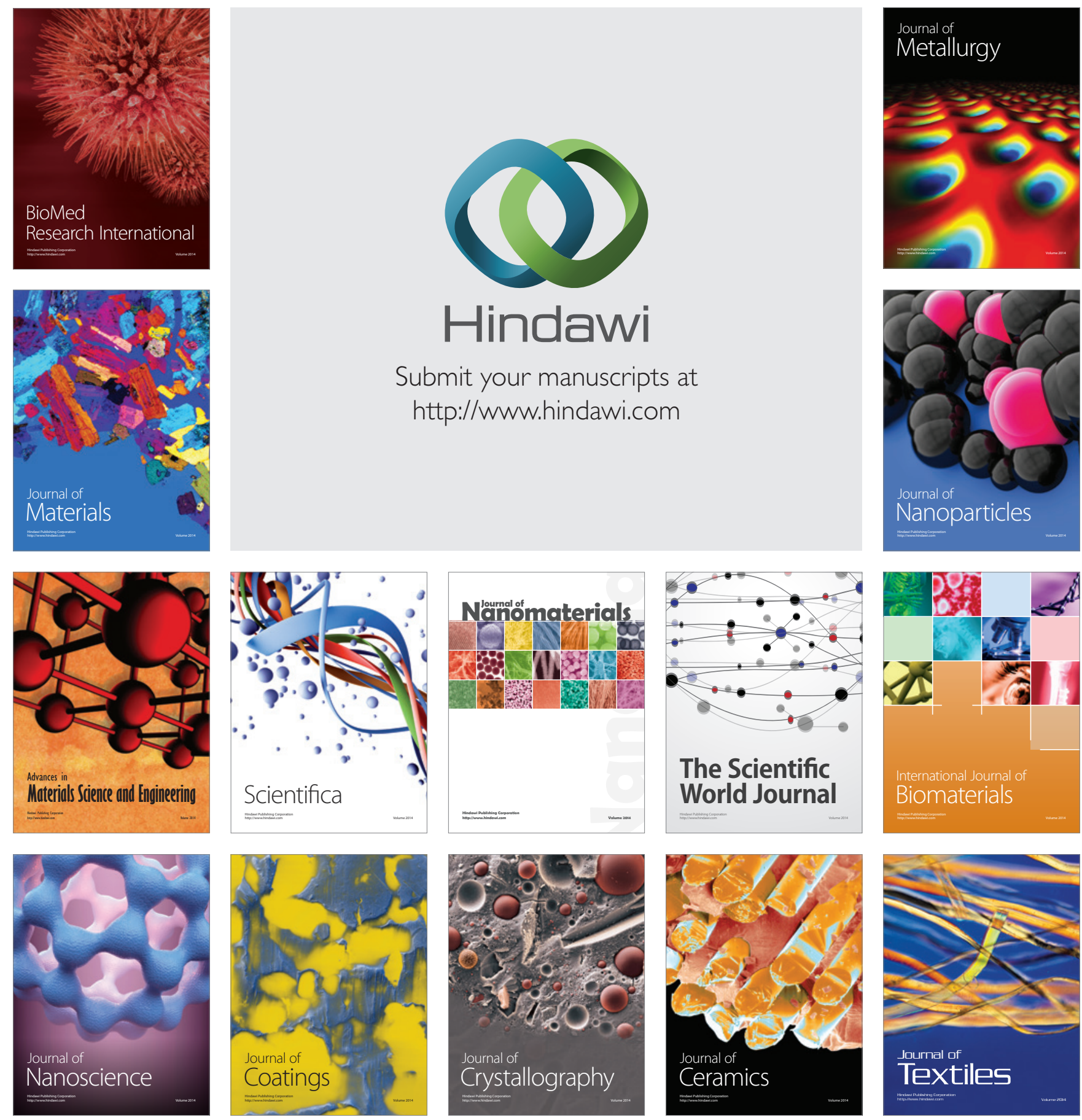